\title{
Impacts of $\mathrm{PM}_{2.5}$ on Ambulatory Blood Pressure Monitoring Indicators Attenuated by Blood Pressure Control Status and Treatment - Two Cities and Two Municipalities, China, 2017-2019
}

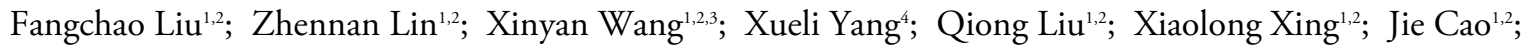 \\ Jianxin Li ${ }^{1,2}$; Keyong Huang ${ }^{1,2}$; Weli Yan'; Tingting $\mathrm{Liu}^{6}$; Wei Li ${ }^{7}$; Shufeng Chen ${ }^{1,2}$; Xiangfeng $\mathrm{Lu}^{1,2}$; \\ Dongfeng $\mathrm{Gu}^{1,2,8}$; Jianfeng Huang ${ }^{1,2,4}$
}

\section{Summary}

What is already known about this topic?

Short-term $\mathrm{PM}_{2.5}$ exposure has been associated with hourly, 24-hour, daytime, and nighttime blood pressure (BP) levels, and further studies focusing whether and how the associations with other ambulatory BP monitoring indicators are warranted. What is added by this report?

This study observed that short-term $\mathrm{PM}_{2.5}$ exposure was associated with BP elevations and was the first to report the associations of short-term $\mathrm{PM}_{2.5}$ exposure with $\mathrm{BP}$ variability. Circadian rhythm of $\mathrm{BP}$ and $\mathrm{BP}$ load among hypertensive patients were found to be modified by controlled BP status or taking angiotensin receptor blockers (ARBs).

What are the implications for public health practice?

This study suggested that antihypertensive therapy, especially with well-controlled BP status may be potential measurements to attenuate adverse impacts of $\mathrm{PM}_{2.5}$ for hypertensive patients with intermediate-tohigh risk of cardiovascular disease (CVD).

High blood pressure (BP) is the leading risk factor for cardiovascular disease (CVD) in China. Previous studies from either lowly or highly polluted regions have revealed that increased fine particulate matter (particles with aerodynamic diameter $\leq 2.5 \mu \mathrm{m} ; \mathrm{PM}_{2.5}$ ) exposure was associated with elevated BP levels and higher risk of hypertension (1). In recent years, using personal portable devices to record individual $\mathrm{PM}_{2.5}$ exposure and ambulatory $\mathrm{BP}$ monitoring (ABPM) to measure BP levels periodically, panel studies also identified the relationship of short-term $\mathrm{PM}_{2.5}$ exposure with hourly, 24-hour, daytime, and nighttime average BP levels (2-3). However, few studies assessed the adverse impacts of $\mathrm{PM}_{2.5}$ with other $\mathrm{ABPM}$ indicators, such as BP variability, circadian rhythm of $\mathrm{BP}$, and BP load, and the modifying effects of BP control status and antihypertensive therapy were also unclear. To clarify these issues, these associations were assessed using a multicity and municipality panel study conducted from 2017 to 2019 in China. BP levels and BP load elevation were associated with short-term $\mathrm{PM}_{2.5}$ exposure. Further stratified analyses demonstrated that the adverse impacts of $\mathrm{PM}_{2.5}$ on $\mathrm{BP}$ variability, circadian rhythm of $\mathrm{BP}$, and $\mathrm{BP}$ load were attenuated among patients with well-controlled BP or taking angiotensin receptor blockers (ARBs), which provided hints about the prevention of $\mathrm{PM}_{2.5}$-related adverse outcomes.

This study used data from a three-phase (winter, summer, spring/autumn) panel study conducted in Beijing, Shanghai, Wuhan, and Xi'an from 2017 to 2019. This panel study recruited participants at intermediate-to-high risk of CVD who were defined as having prehypertension or hypertension combined with at least 1 of the 3 conditions (i.e., central obesity, diabetes mellitus, and dyslipidemia) from the community (2). Questionnaires were administrated to collect demographic characteristics, lifestyle and risk factors, medication uses, etc. Each participant carried a portable monitor to measure real-time individual $\mathrm{PM}_{2.5}$ from the first day to the third day during each phase, and a 24-hour ABPM was scheduled on the third day. This study was approved by the Institutional Review Board of Fuwai Hospital in Beijing, and written informed consent was obtained from all participants before data collection.

This study assessed the association of short-term $\mathrm{PM}_{2.5}$ exposure with several ABPM indicators, including 24-hour, daytime, and nighttime average systolic BP (SBP) and diastolic BP (DBP), SBP and 
DBP variability (i.e., the standard deviation of BP), SBP and DBP load [i.e. the percentage of the counts for $\mathrm{BP}$ readings beyond certain threshold $(130 / 80$ $\mathrm{mmHg}, 135 / 85 \mathrm{mmHg}$, and $120 / 70 \mathrm{mmHg}$ for $24-$ hour, daytime and nighttime, respectively)], and the percentage of nocturnal BP decline [i.e., (daytime BPnighttime $\mathrm{BP}$ )/daytime $\mathrm{BP} \times 100 \%]$. In addition, three methods were used to calculate morning BP surge, including morning BP (i.e., the average BP during the first 2 hours after waking), pre-waking BP surge (i.e., the mean BP during the 2 hours after waking minus the mean BP during the 2 hours before waking), and sleep-trough BP surge (the mean BP during the 2 hours after waking minus the mean $\mathrm{BP}$ of the lowest $\mathrm{BP}$ and the BP before and after the lowest one during sleeping). The exposure windows of $\mathrm{PM}_{2.5}$ included the concurrent day with ABPM (lag0d), lag 1 day (lag1d), lag 2 days (lag2d), and moving average of previous 2 days (MA2d). Estimated changes and 95\% confidence intervals (CIs) with per interquartile range (IQR) increment of $\mathrm{PM}_{2.5}$ exposure $\left(41.96 \mu \mathrm{g} / \mathrm{m}^{3}\right)$ were calculated using a mixed linear model adjusted for age, gender, current alcohol drinking status, ideal level of physical activity, body mass index (BMI), antihypertensive medication uses, diabetes mellitus, dyslipidemia, and natural splines with 3 degrees of freedom for the daily average of personal environmental temperature and relative humidity at the same exposure windows as $\mathrm{PM}_{2.5}$. City-specific and subject-specific random intercepts were used to account for the variations in different cities and withinsubjects correlations. Stratified analysis of BP control status (hypertensive patients with SBP/DBP of less than $140 / 90 \mathrm{mmHg}$ were defined as individuals with controlled BP, otherwise as uncontrolled BP), and for those with uncontrolled $\mathrm{BP}$, a further stratified analysis of ARB uses was conducted. $Z$ tests were conducted to explore the group difference. All analyses were performed using the SAS software (version 9.4; SAS Institute, Cary, NC, USA). A 2-side $P$ of $<0.05$ was demonstrated statistical significance.

After excluding participants attending only one visit, those missing ABPM data or not fulfilling the valid criteria of ABPM data and prehypertensive patients, a total of 277 hypertensive patients with 802 visits were included for analysis. At baseline, the average age was $59.1 \pm 8.5$ years and $113(40.8 \%)$ participants were men. The mean office SBP and DBP were 134.5 $\mathrm{mmHg}$ and $80.4 \mathrm{mmHg}$, respectively. Overall, $91.7 \%$ patients were taking antihypertensive medications, with $33.2 \%$ being taking ARBs (Table 1). Across the three phases, a total of 557 (69.5\%) visits reached wellcontrolled BP status. Among those without controlled BP, $65(26.5 \%)$ visits had been taking ARBs. Generally, short-term $\mathrm{PM}_{2.5}$ exposure was associated with BP levels and BP load; for example, the 24-hour, daytime, and nighttime average SBP increased by $0.68 \mathrm{mmHg}$ (95\% CI: $0.07-1.29), 0.82 \mathrm{mmHg}(95 \%$ CI: $\quad 0.19-1.45)$ and $0.93 \mathrm{mmHg} \quad(95 \% \mathrm{CI}$ : $0.17-1.70)$ per IQR increment of the lag2d $\mathrm{PM}_{2.5}$ concentration (Figure 1A). The 24-hour SBP load and DBP load increased by $1.28 \%(95 \%$ CI: $0.01 \%-2.56 \%$ ) and $1.38 \%$ (95\% CI: $0.22 \%-2.54 \%$ ), respectively, with per IQR increment of the lag1d $\mathrm{PM}_{2.5}$ concentration (Figure 1C). However, shortterm $\mathrm{PM}_{2.5}$ exposure may not be associated with $\mathrm{BP}$ variability, morning $\mathrm{BP}$ surge, or the percentage of nocturnal BP decline (Figure 1B, 1D, 1E). When stratified by BP control status, the increment of 24hour or daytime BP levels associated with $\mathrm{PM}_{2.5}$ exposure tended to be higher among those with uncontrolled BP, but the difference was not statistically significant (Figure 2A). Those with uncontrolled BP were more prone to have higher morning $\mathrm{BP}$ surges than those with controlled BP. For example, per IQR increment of the lag2d $\mathrm{PM}_{2.5}$ concentration was associated with $2.19 \mathrm{mmHg}$ (95\% CI: $0.47-3.91$ ), $1.72 \mathrm{mmHg}$ (95\% CI: $0.20-3.24)$ and $1.47 \mathrm{mmHg}$ (95\% CI: -0.14-3.07) elevation for morning SBP, prewaking SBP surge, and sleep-trough SBP surge, respectively, among patients without controlled BP, while no significant changes were noted in patients with controlled BP (all $P<0.05)$ (Figure 2D). Among patients with uncontrolled $\mathrm{BP}$, the association of $\mathrm{PM}_{2.5}$ exposure with 24-hour, daytime SBP variability was modified by treatment with ARB. For example, 24-hour SBP variability increased by $0.49 \mathrm{mmHg}$ (95\% CI: $-0.22-1.20)$ per IQR increment of lag0d $\mathrm{PM}_{2.5}$ exposure among patients taking ARB, which was significantly higher than those not taking $A R B$ (Figure $2 \mathrm{G}$ ). In addition, patients taking $A R B$ were more likely to have better circadian rhythms for DBP (Figure 2J).

\section{DISCUSSION}

This study observed that short-term $\mathrm{PM}_{2.5}$ exposure was associated with BP elevations and BP load and was the first to report the associations of short-term $\mathrm{PM}_{2.5}$ exposure with BP variability, as well as the circadian rhythm of BP among hypertensive patients being 
TABLE 1. Baseline characteristics of study participants in two cities and two municipalities, China, 2017-2019.

\begin{tabular}{|c|c|c|c|c|c|}
\hline Characteristics & Overall $(n=277)$ & Beijing $(n=64)$ & Shanghai $(n=68)$ & Wuhan $(n=75)$ & Xi'an $(n=70)$ \\
\hline Age, years & $59.1 \pm 8.5$ & $53.5 \pm 8.1$ & $60.3 \pm 8.6$ & $62.6 \pm 6.0$ & $59.3 \pm 8.8$ \\
\hline Male, n (\%) & $113(40.8)$ & $35(54.7)$ & $23(33.8)$ & $23(30.7)$ & $32(45.7)$ \\
\hline $\mathrm{BMI}, \mathrm{kg} / \mathrm{m}^{2}$ & $26.2 \pm 3.2$ & $27.2 \pm 2.6$ & $25.0 \pm 3.0$ & $26.1 \pm 3.5$ & $26.6 \pm 3.2$ \\
\hline Current alcohol drinking status, $\mathrm{n}(\%)$ & $67(24.2)$ & $29(45.3)$ & $11(16.2)$ & $12(16.0)$ & $15(21.4)$ \\
\hline Ideal level of physical activity, n (\%) & $101(36.5)$ & $23(35.9)$ & $14(20.6)$ & $35(46.7)$ & $29(41.4)$ \\
\hline Central obesity, n (\%) & $184(66.4)$ & $53(82.8)$ & $28(41.2)$ & $57(76.0)$ & $46(65.7)$ \\
\hline Office SBP, mmHg & $134.5 \pm 13.6$ & $134.1 \pm 11.4$ & $134.4 \pm 12.0$ & $132.6 \pm 15.4$ & $137.2 \pm 14.7$ \\
\hline Office DBP, mmHg & $80.4 \pm 10.8$ & $83.3 \pm 9.4$ & $79.9 \pm 13.0$ & $76.7 \pm 8.6$ & $82.1 \pm 10.7$ \\
\hline Diabetes mellitus, $\mathrm{n}(\%)$ & $78(28.2)$ & $13(20.3)$ & $29(42.6)$ & $16(21.3)$ & $20(28.6)$ \\
\hline Dyslipidemia, n (\%) & $224(80.9)$ & $54(84.4)$ & $55(80.9)$ & $63(84.0)$ & $52(74.3)$ \\
\hline Antihypertensive drug use, $\mathrm{n}(\%)$ & $254(91.7)$ & $54(84.4)$ & $64(94.1)$ & $70(93.3)$ & $66(94.3)$ \\
\hline ACE inhibitor & $18(6.5)$ & $4(6.3)$ & $2(2.9)$ & $5(6.7)$ & $7(10.0)$ \\
\hline ARB & $92(33.2)$ & $15(23.4)$ & $36(52.9)$ & $27(36.0)$ & $14(20.0)$ \\
\hline$\beta$-receptor blocker & $36(13.0)$ & $14(21.9)$ & $6(8.8)$ & $5(6.7)$ & $11(15.7)$ \\
\hline CCB & $161(58.1)$ & $42(65.6)$ & $30(44.1)$ & $49(65.3)$ & $40(57.1)$ \\
\hline Diuretics & $16(5.8)$ & $2(3.1)$ & $6(8.8)$ & $4(5.3)$ & $4(5.7)$ \\
\hline Others & $13(4.7)$ & $5(7.8)$ & $1(1.5)$ & $2(2.7)$ & $5(7.1)$ \\
\hline One type of medications & $178(64.3)$ & $32(50.0)$ & $48(70.6)$ & $49(65.3)$ & $49(70.0)$ \\
\hline Two types of medications & $61(22.0)$ & $16(25.0)$ & $12(17.6)$ & $18(24.0)$ & $15(21.4)$ \\
\hline Three or more types of medications & $15(5.4)$ & $6(9.4)$ & $4(5.9)$ & $3(4.0)$ & $2(2.9)$ \\
\hline \multicolumn{6}{|l|}{$\mathrm{PM}_{2.5}, \mu \mathrm{g} / \mathrm{m}^{3}$} \\
\hline Lag0d & $50.1 \pm 43.9$ & $49.8 \pm 45.3$ & $33.6 \pm 32.3$ & $57.4 \pm 37.8$ & $57.5 \pm 52.3$ \\
\hline Lag1d & $48.5 \pm 46.0$ & $48.4 \pm 47.9$ & $31.3 \pm 26.0$ & $52.4 \pm 36.6$ & $60.1 \pm 60.4$ \\
\hline Lag2d & $54.2 \pm 50.9$ & $64.6 \pm 66.8$ & $34.0 \pm 34.6$ & $66.6 \pm 45.6$ & $51.8 \pm 47.0$ \\
\hline MA2d & $51.2 \pm 44.6$ & $56.5 \pm 51.0$ & $32.9 \pm 29.0$ & $59.4 \pm 38.2$ & $55.8 \pm 51.7$ \\
\hline \multicolumn{6}{|l|}{ PET, ${ }^{\circ} \mathrm{C}$} \\
\hline Lag0d & $22.3 \pm 5.5$ & $23.8 \pm 3.8$ & $20.9 \pm 6.1$ & $21.8 \pm 7.1$ & $22.5 \pm 4.3$ \\
\hline Lag1d & $22.1 \pm 5.7$ & $23.7 \pm 4.2$ & $21.1 \pm 5.9$ & $21.3 \pm 7.3$ & $22.5 \pm 4.5$ \\
\hline Lag2d & $22.2 \pm 5.6$ & $23.6 \pm 3.8$ & $21.2 \pm 5.8$ & $21.2 \pm 7.3$ & $22.9 \pm 4.2$ \\
\hline MA2d & $22.1 \pm 5.6$ & $23.6 \pm 3.9$ & $21.1 \pm 5.8$ & $21.2 \pm 7.3$ & $22.7 \pm 4.3$ \\
\hline \multicolumn{6}{|l|}{ Relative humidity, $\%$} \\
\hline Lag0d & $53.5 \pm 16.2$ & $40.8 \pm 17.3$ & $66.1 \pm 11.1$ & $59.6 \pm 10.8$ & $49.3 \pm 12.6$ \\
\hline Lag1d & $54.7 \pm 16.0$ & $41.8 \pm 17.3$ & $67.5 \pm 10.6$ & $61.1 \pm 10.0$ & $49.4 \pm 12.0$ \\
\hline Lag2d & $54.1 \pm 15.6$ & $41.6 \pm 17.9$ & $64.7 \pm 7.9$ & $62.7 \pm 8.7$ & $47.2 \pm 12.2$ \\
\hline MA2d & $54.5 \pm 15.4$ & $41.7 \pm 17.3$ & $65.7 \pm 8.6$ & $61.9 \pm 9.0$ & $48.3 \pm 11.8$ \\
\hline
\end{tabular}

Note: Results are presented as mean \pm SD for continuous variables, and frequency (proportion) for categorical variables.

Abbreviations: $\mathrm{SD}=$ standard deviation; $\mathrm{BMI}=$ body mass index; $\mathrm{SBP}=$ systolic blood pressure; $\mathrm{DBP}=$ diastolic blood pressure; $A C E=$ angiotensin-converting enzyme; $A R B=$ angiotensin receptor blockers; $C C B=$ calcium channel blocker; $P M_{2.5}=p a r t i c l e s$ with aerodynamic diameter $\leq 2.5 \mu \mathrm{m}$; PET=personal environmental temperature; lag0d=current day with BP measurement; lag1d=lag of 1 day; lag2d=lag of 2 days; MA2d=the 2-day moving average of $\mathrm{PM}_{2.5}$.

modified by a controlled BP status or by taking ARBs.

BP follows a circadian rhythm with an increase in the morning after waking and a dip during sleep. The existing studies have demonstrated that a disturbed circadian rhythm, including increases of pre-waking BP surges or blunted nocturnal BP declines were associated with increased CVD risk (4). In addition, $\mathrm{BP}$ variability and BP load were also associated with 

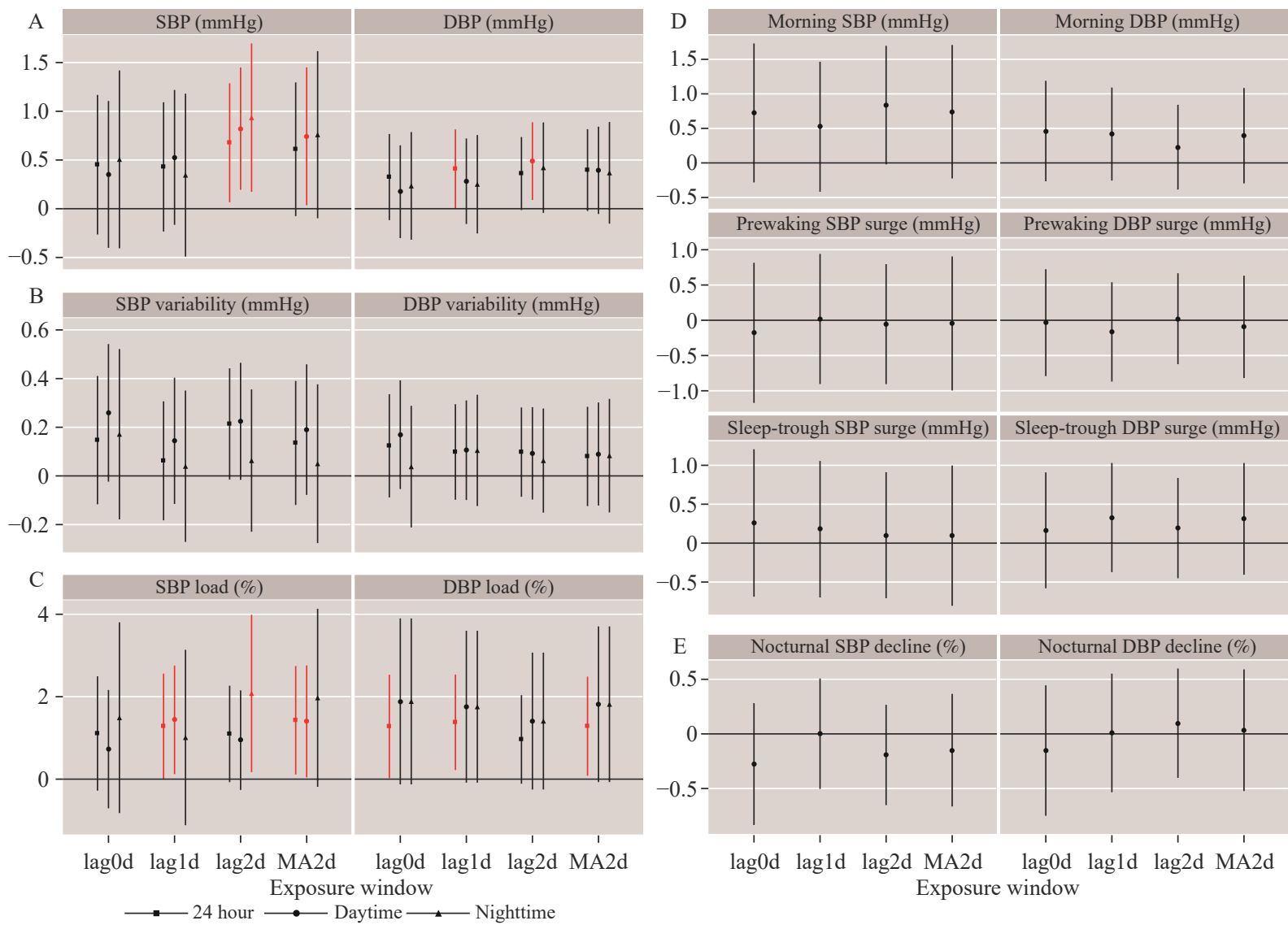

FIGURE 1. Estimated changes and $95 \%$ Cls of BP levels (A), BP variability (B), BP load (C), morning BP surge (D) and the percentage of nocturnal BP decline $(E)$ with per IQR $\left(41.96 \mu \mathrm{g} / \mathrm{m}^{3}\right)$ of $\mathrm{PM}_{2.5}$ exposure among participants in two cities and two municipalities, China, 2017-2019

Note: Statistically significant changes were shown in red.

Abbreviations: $\mathrm{Cl}=$ confidence interval; $\mathrm{BP}=$ blood pressure; IQR=interquartile range; $\mathrm{PM}_{2.5}=$ particles with aerodynamic diameter $\leq 2.5 \mu \mathrm{m}$; $\mathrm{SBP}=$ systolic blood pressure; $\mathrm{DBP}=$ diastolic blood pressure; lag0d=current day with $\mathrm{BP}$ measurement; lag1d=lag of 1 day; lag2d=lag of 2 days; MA2d=the 2-day moving average of $\mathrm{PM}_{2.5}$.

CVD or all-cause mortality (5-6). Up to now, only one study has found that increased short-term $\mathrm{PM}_{10}$ exposure was associated with blunted SBP dipping, and evidence for $\mathrm{PM}_{2.5}$ is still very lacking ( 7 ). Therefore, findings from the current study may provide evidence on the mechanism linking air pollution to increased CVD risk, and potential preventive measurements, such as a well-controlled $\mathrm{BP}$ and taking $\mathrm{ARBs}$, to attenuate the adverse impacts of $\mathrm{PM}_{2.5}$.

Sympathetic nerve activation may play a role in the morning BP surge (8), and nocturnal BP non-dipping may be associated with endothelial dysfunction (9). In accordance with the findings of this study, people reaching well-controlled BP had more stable ANS, hence the adverse impacts of $\mathrm{PM}_{2.5}$ on morning $\mathrm{BP}$ surges were attenuated. On the other hand, exposure to $\mathrm{PM}_{2.5}$ may increase angiotensin II (10), which could induce oxidative stress and inflammation and further promote endothelial dysfunction. ARBs could inhibit the effects of angiotensin II. The potential endothelial dysfunction among patients without taking ARBs interfered the dipping of nocturnal BP. Additionally, the different lag effect trends of $\mathrm{PM}_{2.5}$ on different $\mathrm{BP}$ traits may be due to the various mechanisms of $\mathrm{PM}_{2.5}$ mediated BP elevation, which is an issue worth to exploring in future.

This study firstly reported the association of shortterm $\mathrm{PM}_{2.5}$ exposure with various ABPM indicators, which may provide hints for preventing people from the influences of $\mathrm{PM}_{2.5}$. Additionally, this study was a stringent panel study with a multiphase and multicity design, with individual $\mathrm{PM}_{2.5}$ monitoring and ABPM, which allowed time-dependent covariates to be adjusted, and was less likely to cause misclassification, making the findings more accurate. However, this study was still subject to some limitations. First, owing 

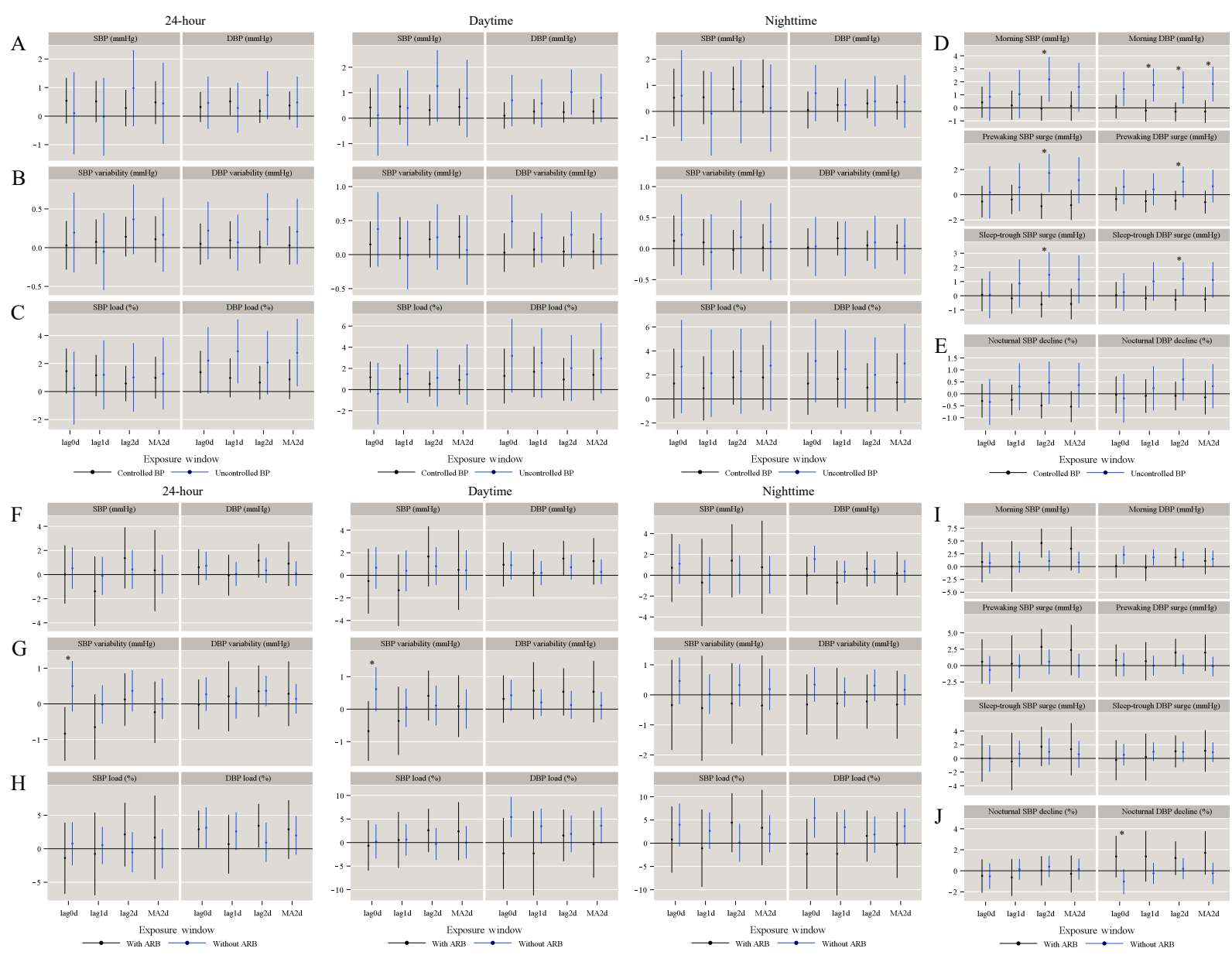

FIGURE 2. Estimated changes and $95 \%$ Cls of BP levels (A, F), BP variability (B, G), BP load (C, H), morning BP surge (D, I) and the percentage of nocturnal BP decline $(E, J)$ with per IQR $\left(41.96 \mu \mathrm{g} / \mathrm{m}^{3}\right)$ of the $\mathrm{PM}_{2.5}$ exposure, stratified by $B P$ control status in overall population and by ARB use in patients without controlled BP in two cities and two municipalities, China, 2017-2019.

${ }^{*} P<0.05$ for differences between two groups.

Abbreviations: $\mathrm{Cl}=$ confidence interval; $\mathrm{BP}=$ blood pressure; $\mathrm{IQR}=$ interquartile range; $\mathrm{PM}_{2.5}=$ particles with aerodynamic diameter $\leq 2.5 \mu \mathrm{m}$; SBP=systolic blood pressure; $\mathrm{DBP}=$ diastolic blood pressure; $\mathrm{ARB}=$ angiotensin receptor blockers; lag0d=current day with BP measurement; lag1d=lag of 1 day; lag2d=lag of 2 days; MA2d=the 2-day moving average of $\mathrm{PM}_{2.5}$.

to the absence of individual gaseous pollutants, the independent effects of $\mathrm{PM}_{2.5}$ have not been assessed. Second, this study used BP measurements from 06:00 to 08:00 instead of BP levels 2 hours after waking to calculate morning surge due to missing information on waking time in this study.

In conclusion, this study added evidence of the associations between short-term $\mathrm{PM}_{2.5}$ exposure and ABPM indicators, and further highlighted antihypertensive therapy, especially with wellcontrolled BP status, which may be a potential measurement to attenuate adverse impacts of $\mathrm{PM}_{2.5}$ for patients with intermediate-to-high risk of CVD.

Funding: The National Key Research and Development Program of China (2016YFC0206503). doi: $10.46234 / \mathrm{ccdcw} 2021.231$

\# Corresponding author: Jianfeng Huang, jianfhuang@sina.com.

\footnotetext{
${ }^{1}$ Department of Epidemiology, Fuwai Hospital, National Center for Cardiovascular Diseases, Chinese Academy of Medical Sciences and Peking Union Medical College, Beijing, China; ${ }^{2}$ Key Laboratory of Cardiovascular Epidemiology, Chinese Academy of Medical Sciences, Beijing, China; ${ }^{3}$ Center for Reproductive Medicine, Tianjin Central Hospital of Gynecology Obstetrics, Tianjin, China; ${ }^{4}$ Tianjin Key Laboratory of Environment, Nutrition and Public Health; Department of Occupational and Environmental Health, School of Public Health, Tianjin Medical University, Tianjin, China; ${ }^{5}$ Department of Clinical Epidemiology \& Clinical Trial Unit, Children's Hospital of Fudan University, National Children's Medical Center, Shanghai, China; ${ }^{6}$ Department of Cardiology, Renmin Hospital of Wuhan University, Wuhan, Hubei, China; ${ }^{7}$ Function Test Center, Fuwai Hospital, National Center for Cardiovascular Diseases, Chinese Academy of Medical Sciences and Peking Union Medical College, Beijing, China;

${ }^{8}$ School of Medicine, Southern University of Science and Technology, Shenzhen, Guangdong, China.
} 
Submitted: October 25, 2021; Accepted: November 03, 2021

\section{REFERENCES}

1. Yang BY, Qian ZM, Howard SW, Vaughn MG, Fan SJ, Liu KK, et al. Global association between ambient air pollution and blood pressure: a systematic review and meta-analysis. Environ Pollut 2018;235:57688. http://dx.doi.org/10.1016/j.envpol.2018.01.001.

2. Lin ZN, Wang XY, Liu FC, Yang XL, Liu Q, Xing XL, et al. Impacts of short-term fine particulate matter exposure on blood pressure were modified by control status and treatment in hypertensive patients. Hypertension 2021;78(1):174 - 83. http://dx.doi.org/10.1161/HYPE RTENSIONAHA.120.16611.

3. Santos UP, Ferreira Braga AL, Bueno Garcia ML, Amador Pereira LA, Lin CA, Chiarelli PS, et al. Exposure to fine particles increases blood pressure of hypertensive outdoor workers: a panel study. Environ Res 2019;174:88 - 94. http://dx.doi.org/10.1016/j.envres.2019.04.021.

4. Zhang JY, Sun RY, Jiang TT, Yang GR, Chen LH. Circadian blood pressure rhythm in cardiovascular and renal health and disease. Biomolecules 2021;11(6):868. http://dx.doi.org/10.3390/biom 11060868

5. Stevens SL, Wood S, Koshiaris C, Law K, Glasziou P, Stevens RJ, et al. Blood pressure variability and cardiovascular disease: systematic review and meta-analysis. BMJ 2016;354:i4098. http://dx.doi.org/10.1136/ bmj.i4098.

6. Li Y, Thijs L, Boggia J, Asayama K, Hansen TW, Kikuya M, et al. Blood pressure load does not add to ambulatory blood pressure level for cardiovascular risk stratification. Hypertension 2014;63(5):925 - 33. http://dx.doi.org/10.1161/HYPERTENSIONAHA.113.02780.

7. Tsai DH, Riediker M, Wuerzner G, Maillard M, Marques-Vidal P, Paccaud F, et al. Short-term increase in particulate matter blunts nocturnal blood pressure dipping and daytime urinary sodium excretion. Hypertension 2012;60(4):1061 - 9. http://dx.doi.org/10. 1161/HYPERTENSIONAHA.112.195370.

8. Lambert EA, Chatzivlastou K, Schlaich M, Lambert G, Head GA. Morning surge in blood pressure is associated with reactivity of the sympathetic nervous system. Am J Hypertens 2014;27(6):783 - 92. http://dx.doi.org/10.1093/ajh/hpt273.

9. Chang JC, Xiao R, Meyers KE, Mercer-Rosa L, Natarajan SS, Weiss $\mathrm{PF}$, et al. Nocturnal blood pressure dipping as a marker of endothelial function and subclinical atherosclerosis in pediatric-onset systemic lupus erythematosus. Arthritis Res Ther 2020;22(1):129. http://dx.doi.org/ 10.1186/s13075-020-02224-w.

10. Ghelfi E, Wellenius GA, Lawrence J, Millet E, Gonzalez-Flecha B. Cardiac oxidative stress and dysfunction by fine concentrated ambient particles (CAPs) are mediated by angiotensin-II. Inhal Toxicol 2010;22(11):963 - 72. http://dx.doi.org/10.3109/08958378.2010. 503322. 